\title{
EXPOSITION OF VARIOUS POTENTIAL RESEARCH TOPICS
}

\section{PETERBISSONNET}

Independent Researcher, Fusagasuga, Colombia, South America

\begin{abstract}
The intent of this paper is to present hopefully new and fruitful topic areas for current and future research. 1. Aredark matter and dark energy really caused by strange new particles or something else? 2. Normal surface geometry in differential geometry is built upon what is called the Symmetric Coefficient of the Second Fundamental Form. Is there such a thing as the Asymmetric Coefficient of the Second Fundamental Form? 3. Is it possible to have one equation which will give the basic intrinsic spin values? 4. Is it possible to derive a generalized transport flux using the Fokker-Planck equation? 5. Does the weak interaction harbor a powerful energy source? 6. Could the predicted fading of the earth's magnetic field lead to another ice age? What really is lightning?

KEYWORDS:Dark Energy, Dark Matter, An Asymmetric Coefficient of the Second Fundamental Form, A Common Source of Basic Intrinsic Spin Values, Generalized Transport Flux, The Powerful Energy Source of the Weak Interaction, Fading and Contracting of Earth's Magnetic Field \& Lightning
\end{abstract}

Received: Jul 04, 2018; Accepted: Jul 24, 2018; Published: Aug 13, 2018; Paper Id.: IJPRDEC20181

\section{INTRODUCTION}

Theoretical research is fun!Why is it fun?Because it only requires a paper, pencil, and a willing and inquiring mind.It is convenient and can be done anywhere and at anytime and does not require expensive machinery or equipment, consequently, it is very economical.

\section{ON THE POSSIBILITY THAT DARK MATTER AND DARK ENERGY ARE REALLY GEOMETRIC IN NATURE ${ }^{1}$}

It is supposed that the coefficient of connection $\Gamma_{i k}^{r}$ on the galactic scale can be written as

$$
-\Gamma_{i k}^{r}=A_{i k} \bar{\alpha}^{r}-\left\{\begin{array}{c}
r \\
i k
\end{array}\right\}
$$

where $\left\{\begin{array}{l}r \\ i k\end{array}\right\}$ is considered to be the Christoffel symbol, $A_{i k}$ is the symmetric coefficient of the second fundamental form in differential geometry and $\bar{\alpha}^{r}$ would be considered to be some sort of small vector average (a free vector) over the large region in question, in this case a galaxy.In the case of a galaxy, which might be considered to be an "umbilical", "navel", or "dimple" point in space-time, due to their central black hole causing tremendous deformations in space-time, we can consider that

$$
A_{i k}=\kappa g_{i k}
$$

Where $g_{i k}$ is the space time metric, where $\kappa$ is a curvature constant having the dimensions of an inverse 
distance and being dependent upon the galaxy.Thus, our equation becomes

$$
-\Gamma_{i k}^{j}=\kappa g_{i k} \bar{\alpha}^{j}-\left\{\begin{array}{c}
j \\
i k
\end{array}\right\}=\kappa g_{i k}-\frac{1}{2} g^{r j}\left(g_{i r / k}-g_{i k / r}+g_{r k / i}\right)
$$

Using a weak field and non-relativistic velocity approximation, we know that

$$
-\Gamma_{00}^{j}=\kappa g_{00} \bar{\alpha}^{j}-\left\{\begin{array}{l}
j \\
00
\end{array}\right\} \text { where } g_{00}=g_{00 \text { Lorentz }}+\varepsilon \gamma_{00} \text {, where } \mathcal{E} \text { is a small constant.We also assume that the }
$$
metric has the signature of (-1-1-1+1) in a Lorentzian space.

$$
\begin{aligned}
& \left\{\begin{array}{l}
j \\
00
\end{array}\right\} \approx \frac{1}{2} \varepsilon \gamma_{00 / j} \\
& -\Gamma_{00}^{j}=\kappa\left(1+\varepsilon \gamma_{00}\right) \bar{\alpha}^{j}-\frac{1}{2} \varepsilon \gamma_{00 / j}
\end{aligned}
$$

Looking only at $\mathrm{j}=1$ corresponding to the $\mathrm{r}$ coordinate in a spherical coordinate system. We know that in the limit of weak gravitational fields and small velocities that $\varepsilon \gamma_{00}=\frac{2 \phi}{c^{2}}$ where $\phi=-G_{0} \frac{M}{r}$ and further $\varepsilon \gamma_{00 / 1}=\frac{2 G_{0}}{c^{2}} \frac{M}{r^{2}}$ so that

$$
-c^{2} \Gamma_{00}^{1}=c^{2} \kappa \bar{\alpha}^{1}-2 \kappa \bar{\alpha}^{1} G_{0} \frac{M}{r}-G_{0} \frac{M}{r^{2}}
$$

The $\bar{\alpha}^{1}$ components are postulated to be very small and necessarily of such a magnitude as to not be of any consequence on a planetary or solar system scale in order to be consistent with the experimental observation of the validity of Newtonian gravitation on such scales.It is postulated that these $\bar{\alpha}^{1}$ exist, but as to what they actually represent physically is unknown. When, $\bar{\alpha}^{1} \neq 0$, we thus see a modification to the Newtonian $\left(\mathrm{r}^{-2}\right)$ forces, as another hybrid $\left(\mathrm{r}^{-1}\right)$ acceleration, partly of gravitational origin and partly of non-gravitational origin comes into play.As both of the $r^{-1}$ and $r^{-2}$ forces die off with increased distance, there remains a residual acceleration of $c^{2} \kappa \bar{\alpha}^{1}$ at work.If this, $\kappa \bar{\alpha}^{1}>0$, then this residual force is repulsive and the $\mathrm{r}^{-1}$ force is attractive.This residual repulsive force may cause a volume of galaxies to repel each other, thus causing the volume to expand or inflate.Could this first term in equation 5 above then be the source of dark energy ${ }^{2}$, while the second term would be the source of dark matter, while the last term would be the usual attractive Newtonianforce.The reason for designating the second term as the source of dark matter ${ }^{3}$ is due to the following argument.Consider a small piece of matter $\mathrm{dm}$ in the second term at a distance $\mathrm{r}$ and equate this force with the centripetal force

$$
2 \kappa \bar{\alpha}^{1} G_{0} \frac{d m}{r}=\frac{V^{2} d m}{r}
$$

From both sides, the $\mathrm{dm}$ and $\mathrm{r}^{-1}$ terms cancel out giving the velocity $\mathrm{V}^{2}$ as a constant across the galaxy dimensions, which is typical of the dark matter hypothesis. 


\section{THE ASYMMETRIC COEFFICIENT OF THE SECOND FUNDAMENTAL FORM IN DIFFERENTIAL GEOMETRY ${ }^{1}$}

These asymmetric coefficients were discovered by the author in 1972 at a university that the author is embarrassed and ashamed to ever have attended.Nevertheless, the author feels that, in spite of his paper using these coefficients, that there ought to be other applications.Equation 6 below is the Gauss equation and equation 7 is the Weingarten equation.

$$
\begin{aligned}
& X_{/ i / k}=\Gamma_{i k}^{r} X_{/ r}+A_{i k} N \\
& N_{/ j}=-A_{j}^{r} X_{/ r} \\
& X_{/ i} \bullet X_{/ j}=g_{i j} \\
& X_{/ i} \bullet N=0 \\
& N \bullet N=1
\end{aligned}
$$

$\mathrm{X}$ is a position vector to a point on a hypersurface.Its derivatives $X_{/ i}$ are tangent vectors to the hypersurface at a point and $\mathrm{N}$ is a normal vector to the hypersurface at that point. $\Gamma_{i k}^{r}$ represent the symmetric coefficient of connections, $A_{i k}$ represent the symmetric coefficients of the Second Fundamental Form, $g_{i j}$ represent the symmetric coefficient of the First Fundamental Form or also known as the metric.The above equations result in the usual development of Riemannian geometry and of General Relativity.The author discovered a basis for a new postulated geometry in 1972, given by the following equations.

$$
\begin{aligned}
& X_{/ i / k}=\Gamma_{i k}^{r} X_{/ r}+A_{i k} N \\
& N_{/ j}=-B_{j}^{r} X_{/ r}+d_{j} N \\
& X_{/ i} \bullet X_{/ j}=g_{i j} \\
& X_{/ i} \bullet N=\alpha_{i} \\
& N \bullet N=\gamma^{2}
\end{aligned}
$$

Where $\alpha_{i}$ and $\gamma_{\text {are small. }} B_{i k}$ arethe asymmetric coefficient of the second fundamental form. The author postulated these equations in his paper "Is Intrinsic Spin Really a Quantum Mechanical Concept?" and has given all the details of his calculations in that paper (please see the reference section).The author, however, feels that these equations can also be used in other applications, and, therefore, presents them to others who may find new research applications for them.

\section{POSSIBLE COMMONALITY OF SOURCE OF BASIC INTRINSIC SPIN VALUES ${ }^{4}$}

The following formula seems to indicate a possible commonality of the source for basic intrinsic spin values.

$$
\begin{aligned}
& S(x)=2^{\cos x}[\cos 2 x]^{2} \\
& x=\frac{n \pi}{4}
\end{aligned}
$$


Table 1: Calculation of Basic Spin Values

\begin{tabular}{|c|c|c|c|c|l|}
\hline $\mathbf{S}(\mathbf{x})$ & $\mathbf{x}=\mathbf{n} \boldsymbol{\pi} / \mathbf{4}$ & $\mathbf{n}$ & Radians & Value & Particle \\
\hline 2 & 0 & 0 & 0 & 2 & graviton \\
\hline 0 & 0.7854 & 1 & $\pi / 4$ & 0 & boson \\
\hline 1 & 1.5708 & 2 & $\pi / 2$ & 1 & boson \\
\hline 0 & 2.3562 & 3 & $3 \pi / 4$ & 0 & boson \\
\hline 0.5 & 3.1416 & 4 & $\pi$ & $1 / 2$ & fermion \\
\hline 0 & 3.927 & 5 & $5 \pi / 4$ & 0 & \\
\hline 1 & 4.7124 & 6 & $3 \pi / 2$ & 1 & \\
\hline 0 & 5.4978 & 7 & $7 \pi / 4$ & 0 & \\
\hline 2 & 6.2832 & 8 & $2 \pi$ & 2 & \\
\hline
\end{tabular}

If this is a valid formula, could there also be a similar formula for charge values, possibly based upon the number 3 ?

\section{DERIVATION OF A TRANSPORT FLUX FROM THE FOKKER-PLANCK EQUATION ${ }^{5}$}

The derivation used will be that of C. Kittel (and his source) ${ }^{6}$.The derivation derives from the Smoluchowski equation using a conditional probability $\mathrm{P}(\mathrm{z} \mid \mathrm{y}, \mathrm{t})$ that a particle at $\mathrm{z}$ at $\mathrm{t}=0$ will be at $\mathrm{y}$ during the time interval $\Delta t$.The result derived is the Fokker-Planck equation without sources.

$$
\frac{\partial P}{\partial t}+\frac{\partial[A(y) P]}{\partial y}-\frac{1}{2} \frac{\partial^{2}[B(y) P]}{\partial y^{2}}=0
$$

If $\mathrm{A}(\mathrm{y})=0$, then there are equal probabilities of moving either left or right.If $\mathrm{B}(\mathrm{y})$ is independent of position or, in other words, there is anisotropic environment, then this reduces to the usual diffusion equation.Now, let's construct the covariant formulation utilizing the symbolism that $/_{\mu}$ is a covariant partial derivative ${ }^{7}$ with respect to the co-ordinate $\mu$, while $/^{\mu}$ represents the contravariant partial derivative.

$$
\left(A^{\mu} P\right)_{/ \mu}=\nabla \cdot(\mathbf{A} P)+\frac{\partial\left(A^{4} P\right)}{\partial(c t)}=\left(A^{1} P\right)_{/ 1}+\left(A^{2} P\right)_{/ 2}+\left(A^{3} P\right)_{/ 3}+\left(A^{4} P\right)_{/ 4}
$$

This equation then incorporates the first two terms on the left of equation 8 .

$$
\begin{aligned}
& \nabla^{2}(B P)=\nabla \cdot \nabla(B P) \text { which can be represented covariantly as }(B P)_{/ \mu}^{/ \mu} \\
& \left(A^{\mu} P\right)_{/ \mu}+(B P)^{/ \mu} / \mu=S=\text { source/sink (10) }
\end{aligned}
$$

This is still not in completely covariant form since we have not used the covariant derivative // which involves the Christoffel symbols in curved coordinate systems. When we do this, we obtain the following: 


$$
\left(A^{\mu} P\right)_{\| \mu}+(B P)_{\| \mu}^{\prime \mu}=S
$$

Which is now a covariant equation. We can now factor out the covariant derivative and obtain:

$$
\left[\left(A^{\mu} P\right)+(B P)^{/ \mu}\right]_{/ / \mu}=S=J_{/ / \mu}^{\mu}
$$

$J^{\mu}=\left(A^{\mu} P\right)+(B P)^{\prime \mu}=$ transports flux or current density.This is slightly more general than other transport fluxes usually found and represented in irreversible thermodynamics, such as (again from Kittel) ${ }^{6,8}$ :

$\mathrm{J}_{\mathrm{x}} \mathrm{K}_{\mathrm{x}}=-\nabla \mathrm{W}$

Ohms law: $\mathrm{x}=\mathrm{e}$ and $\mathrm{K}_{\mathrm{e}}=$ electrical conductivity, $\mathrm{W}=$ electric potential, $\mathrm{J}_{\mathrm{e}}=$ current density

Fourier's law: $\mathrm{x}=\mathrm{q}$ and $\mathrm{K}_{\mathrm{q}}=$ thermal conductivity, $\mathrm{W}=$ Temperature, $\mathrm{J}_{\mathrm{q}}=$ heat current density

Fick's law: $\mathrm{x}=\mathrm{m}$ and $\mathrm{K}_{\mathrm{m}}=$ diffusivity, $\mathrm{W}=$ particle or mass concentration, $\mathrm{J}_{\mathrm{m}}=$ mass or particle current density.

$$
J_{\mu}=\left(A_{\mu} P\right)+(B P)_{/ \mu}
$$

It is to be noted that equation 13 is somewhat similar to the Ginzburg-Landau equation from superconductivity'

$J_{s}=\frac{e^{*}|\psi|^{2}}{m^{*}}\left(\hbar \nabla \varphi-\frac{e^{*}}{c} \mathbf{A}\right)$, where $\mathrm{e}^{*}=2 \mathrm{e}$ and $\mathrm{m}^{*}=2 \mathrm{~m}$ for electron pairs.It would be interesting to know how many other venues this structural form for transport flux arises, and what are, if any, the common characteristics they all share.

\section{IS THE WEAK INTERACTION HARBORING A POWERFUL ENERGY SOURCE? ${ }^{10}$}

Just what is the plausible physical nature of the weak interaction?This endeavor starts out by noticing the similarity in values of two particular numbers. We begin by giving the following sets of values which will be used in the calculation of those two numbers.

$$
\begin{aligned}
& \mathrm{M}_{\mathrm{w}}=\text { considered to be the average boson mass of the weak force }=91 \mathrm{~m}_{\mathrm{p}} \\
& \mathrm{m}_{\mathrm{p}}=\text { mass of the proton }=1.673 \times 10^{-24} \mathrm{~g} \\
& \mathrm{~m}_{\mathrm{e}}=\text { mass of the electron }=9.109 \times 10^{-28} \mathrm{~g} \\
& \mathrm{~h}=\text { Planck's constant }=6.626 \times 10^{-27} \mathrm{erg} \mathrm{sec} \\
& \mathrm{G}_{\mathrm{o}}=\text { gravitational constant }=6.670 \times 10^{-8} \text { dyne } \mathrm{cm}^{2} \mathrm{~g}^{-2} \\
& \mathrm{r}_{\mathrm{Go}}=\left(\mathrm{G}_{\mathrm{o}} \mathrm{h} / \mathrm{c}^{3}\right)^{1 / 2} \mathrm{~cm} \\
& \mathrm{R}_{\mathrm{oA}}=\text { a value, which is assumed to be comparable to the range of the weak force }=\sim 10^{-17} \mathrm{~cm} \\
& \mathrm{c}=\text { speed of light }=3 \times 10^{10} \mathrm{~cm} \mathrm{sec}^{-1}
\end{aligned}
$$


$\mathrm{m}_{\mathrm{p}} / \mathrm{m}_{\mathrm{e}}=1836.12$

The first number $\mathrm{N}_{1}$ to be calculated is

$$
\begin{aligned}
& \mathrm{N}_{1}=\mathrm{r}_{\mathrm{Go}} \mathrm{M}_{\mathrm{w}}^{3} \mathrm{~cm} \mathrm{~g}^{3} \\
& \text { wherer }_{\mathrm{Go}}=\left(\mathrm{G}_{\mathrm{o}} \mathrm{h} / \mathrm{c}^{3}\right)^{1 / 2} \mathrm{~cm} \\
& \mathrm{r}_{\mathrm{Go}} \mathrm{M}_{\mathrm{w}}{ }^{3}=\left[6.670 \times 10^{-8} \text { dyne } \mathrm{cm}^{2} \mathrm{~g}^{-2} \times 6.626 \times 10^{-27} \mathrm{erg} \mathrm{sec} /\left(3 \times 10^{10} \mathrm{~cm} \mathrm{sec}^{-1}\right)^{3}\right]^{1 / 2}\left[91 \times 1.673 \times 10^{-24} \mathrm{~g}\right]^{3} \\
& \mathrm{r}_{\mathrm{Go}} \mathrm{M}_{\mathrm{w}}{ }^{3}=1.428 \times 10^{-98} \mathrm{~cm} \mathrm{~g}^{3}
\end{aligned}
$$

The second number $\mathrm{N}_{2}$ to be calculated is

$$
\begin{aligned}
& \mathrm{N}_{2}=\mathrm{R}_{\mathrm{oA}} \mathrm{m}_{\mathrm{e}}^{3}=\left(10^{-17} \mathrm{~cm}\right)\left(9.109 \times 10^{-28} \mathrm{~g}\right)^{3} \\
& \mathrm{R}_{\mathrm{oA}} \mathrm{m}_{\mathrm{e}}^{3}=0.756 \times 10^{-98} \mathrm{~cm} \mathrm{~g}^{3}
\end{aligned}
$$

It is extremely coincidental that these two numbers are that close. The hypothesis of this paper is that these are, in reality, an equivalence, viz. $\mathrm{N}_{1}=\mathrm{N}_{2}$ or

$$
\mathrm{r}_{\mathrm{Go}} \mathrm{M}_{\mathrm{w}}{ }^{3}=\mathrm{R}_{\mathrm{oC}} \mathrm{m}_{\mathrm{e}}{ }^{3}
$$

When this assumption is made, a calculated value $\mathrm{R}_{\mathrm{oC}}$ from this postulated equivalence is

$$
\begin{aligned}
& \mathrm{R}_{\mathrm{oC}}=\mathrm{r}_{\mathrm{Go}} \mathrm{M}_{\mathrm{w}}^{3} / \mathrm{m}_{\mathrm{e}}^{3}=1.428 \times 10^{-98} \mathrm{~cm} \mathrm{~g}^{3} /\left(9.109 \times 10^{-28} \mathrm{~g}\right)^{3} \text { or } \\
& \mathrm{R}_{\mathrm{oC}}=1.889 \times 10^{-17} \mathrm{~cm} \text {, which is roughly ten times less than the value calculated from the uncertainty principle for }
\end{aligned}
$$
the vector boson of $91 \mathrm{~m}_{\mathrm{p}}$.

Could this particular set of dimensions, $\mathrm{cm} \mathrm{g}^{3}$, be especially predisposed to a relationship between the weak interaction and the

gravitational 'constant'?We can explore this possibility further by attempting to create a simple non-quantum mechanical modelof the weak interaction in order to determine its true nature.

To this end, let the model begin with a tiny variable electric dipole according to the following equation $\mathrm{p}=\mathrm{q}\left(\mathrm{R}_{\mathrm{oC}}-\mathrm{R}\right)$ esu cm, where $\mathrm{q}=(2 \mathrm{hc})^{1 / 2} \mathrm{esu}, \mathrm{R}_{\mathrm{oC}}=\mathrm{r}_{\mathrm{Go}} \mathrm{M}_{\mathrm{w}}{ }^{3} / \mathrm{m}_{\mathrm{e}}{ }^{3}$

$\mathrm{R}=\mathrm{r}_{\mathrm{G}} \mathrm{M}_{\mathrm{w}}{ }^{3} / \mathrm{m}_{\mathrm{e}}{ }^{3}$, where $\mathrm{r}_{\mathrm{G}}=\left(\mathrm{G} \mathrm{h} / \mathrm{c}^{3}\right)^{1 / 2}$, Gnow considered to be a real scalar variable for computational purposes.

We then form the square of $\mathrm{p}$,

$\mathrm{p}^{2}=\mathrm{q}^{2}\left(\mathrm{R}_{\mathrm{oC}}-\mathrm{R}\right)^{2} \mathrm{esu}^{2} \mathrm{~cm}^{2}$, noting that $\mathrm{esu}^{2} \mathrm{~cm}^{2}=\operatorname{erg~} \mathrm{cm}^{3}$.

We now posit that the weak interaction is activated by the variable $G \rightarrow 0$.Then we can take the limit

$$
\begin{aligned}
& \underset{G \rightarrow 0}{\operatorname{Limit}} p^{2}=q^{2} R_{0} c^{2} \\
& \mathrm{q}^{2} \mathrm{R}_{\mathrm{oC}}{ }^{2}=(2 \mathrm{hc})\left(1.889 \times 10^{-17} \mathrm{~cm}\right)^{2}= \\
& \left(2 \times 6.626 \times 10^{-27} \mathrm{erg} \mathrm{sec} \times 3 \times 10^{10} \mathrm{~cm} \mathrm{sec}^{-1}\right)\left(3.568 \times 10^{-34} \mathrm{~cm}^{2}\right) \\
& \mathrm{q}^{2} \mathrm{R}_{\mathrm{oC}}{ }^{2}=1.419 \times 10^{-49} \mathrm{erg} \mathrm{cm}^{3} .
\end{aligned}
$$


In the 1960's, the Fermi weak interaction constant was accepted as $1.41 \times 10^{-49} \mathrm{erg} \mathrm{cm}^{3}$ so that this simplistic model is somewhat instructive.Today, it is $1.43 \times 10^{-49} \mathrm{erg} \mathrm{cm}^{3}$. What the above hopefully show is the possibility of a relationship between a variable gravitational 'constant' and the weak interaction. Assuming that this is the case, how could this assertion be verified experimentally?One way is by calculating (using the uncertainty principle) the mass associated with the value $\mathrm{R}_{\mathrm{oC}}=1.889 \times 10^{-17} \mathrm{~cm}$. This value is $\mathrm{M}_{\mathrm{oc}}=1118 \mathrm{~m}_{\mathrm{p}}$. If this model is correct, then CERN should find a new particle at around 1118 times the mass of the proton. Just to be fair, other authors have tackled similar issues; however, not utilizing the concept of a variable gravitational 'constant'.For example, B.H. Lavenda ${ }^{11}$ has calculated, on totally different grounds using string theory, a particle of 1000 times the mass of the proton associated with the weak interaction. The author hopes that his value will be the more exact one thus verifying a variable gravitational 'constant' associated with the weak interaction.

\section{LIGHTNING AND THE EARTH'S CHANGING MAGNETIC FIELD ${ }^{12}$}

The current train of thought in earth physics seems to be that the earth's magnetic field is going to reverse its poles at some point, which evidence from hardened lava suggests happens every few hundred thousand years.Apparently, the earth's magnetic field fades slowly and then reappears with its poles reversed.The main question for this paper is how slowly does the magnetic field fade?The current view is that when the earth's magnetic field disappears, this aegis will no longer protect us from charged radiation from the sun, as well as from charged cosmic rays.Such protection is evidenced from the existence of the Van Allen Belts.

However, before this complete fading out occurs, there is another possibility, which could suggest itself; namely, the magnetic field could very slowly contract as part of its disappearance.As this field contracts, there is a distinct possibility that it could drag the Van Allen Belts much closer to the earth and to the earth's atmosphere, thereby interacting with the earth's atmosphere. What would happen if a large source of 'free charged particles' is brought into contact with the earth's atmosphere?

The current view seems to be that lightning in thunderstorms is probably caused by ice particles interacting with each other through friction, thus causing charge separation, although there seems to be no absolute consensus for this, considering the effects of cosmic rays ${ }^{13}$. Of course, there are other causes of lightning, caused by frictional charge separation, such as is found around erupting volcanoes and near tornadoes. This paper wishes to point out that the interaction of the atmosphere with this large source of hypothetically free plasma found in the Van Allen belts could result in larger and more devastating storms with lightning bolts many times more powerful than those we now experience.That is why the question was asked earlier as to how slowly the magnetic field would fade.If it fades over a very long time, such as decades or hundreds of years, while the contraction stabilizes, then as the charged particles from the Van Allen Belts are depleted into the atmosphere, there is a possibility of a continued renewal of charged particles from the sun and from cosmic radiation. We might then end up with a steady state process between depletion and renewal of charged particles in the Van Allen Belts during its period of interaction with the earth's atmosphere.

The hypothetically much more powerful lightning bolts which might ensue could, in effect, heat up the atmosphere, as well as the earth itself, causing a much faster evaporation of moisture.Ice ages cannot occur unless a part of the earth is hot enough to create the evaporative moisture which then condenses over the cooler parts of the earth.The Milankovitch Theory ${ }^{14,15}$ and other theories give astronomical causes for ice ages, but these theories are apparently not 
entirely in accord with all the evidence.Perhaps there are non-astronomical or terrestrial contributions to the theory of ice ages, such as might be possibly described by this paper.While this paper assumed powerful lightning bolts as flows of electrons, there are other charged particles in the Van Allen Belts.Could there ever be proton lightning bolts?Incidentally, what really is the source of the usual lightning in theatmosphere. The author would like to put in his one and one-half cent theory, but please don't laugh too hard.Well here goes, the water molecule is like a tiny battery, right? What if, like the long handle on your flashlight with the batteries in series, the water molecules line up and create long strands or cables, and like the cables holding up a bridge, they band together somehow into these even larger cables like the bridge cables.They become like giant flexible batteries with potential differences of hundreds of thousands of volts.Suppose further that these giant cables are flailing around and happen to create a torus-like structure and what happens?They short out causing a tremendous spark or lightning. These giant cables could also be formed vertically or horizontally in either configuration, such as positive to negative or negative to positive accounting for the cloud to ground, ground to cloud, or cloud to cloud lightning.Cosmic rays could also affect the ionization tracks through these cables causing them, unlike flashlight batteries, to short out.How could all of this take place?Possibly there are many contributing factors like the magnetic field of the earth, the electric fields from the Van Allen belts, and in addition to the quantum mechanics of large-scale systems of molecules in which stable configurations are momentarily created. The author even thinks that these cables might even be a fifth state of matter lying in between the plasma state and the gaseous state!Not bad for one and one-half cents!

\section{CONCLUSIONS}

This paper has attempted to give exposition to some possible new areas of exciting theoretical research that might prove fruitful if pursued with open-mindedness.Some of these concepts are not only on the cutting edge they might be entirely outside of the bounds of normally accepted research.There is no law against that! Innovation in research these days, sometimes means having the courage to charge ahead, and if that means leaving the normally accepted channels in the search for truth, then so be it!

\section{REFERENCES}

1. Bissonnet, P. (2005). Is Intrinsic Spin Really a Quantum Mechanical Concept? Hadronic Journal, 28, 567-584.See also http://vixra.org/abs/1606.0311

2. Dark Matter, Wikipedia. Retrieved from https://en.wikipedia.org/wiki/Dark_matter

3. Dark Energy, Wikipedia. Retrieved from https://en.wikipedia.org/wiki/Dark_energy

4. Bissonnet, P. (2016). Notation on the Possible Commonality of Source of Basic Intrinsic Spin Values. Retrieved from http://vixra.org/abs/1610.0037

5. Bissonnet, P. (2017).Derivation of a Transport Flux from the Fokker-Planck Equation.Retrieved from http://vixra.org/abs/1706.0545

6. Charles Kittel (1964).Elementary Statistical Physics, John Wiley and Sons, Inc., New York, p. 157 (Third Printing)Kittel's reference is:Ming Chen Wang and G.E. Uhlenbeck (1945) Revs. Mod. Phys. 17, 331.

7. R. Adler, M. Bazin, M. Schiffer (1965).Introduction to General Relativity, McGraw-Hill.

8. G.G. Koerber, (1962).Properties of Solids, Prentice-Hall, Inc., Englewood Cliffs, New Jersey, p. 13. 
9. Beasley, M.R. (2009) Notes on the Ginzburg-Landau Theory, ICMR Summer School on Novel Superconductors University of California, Santa Barbara Aug 2 - Aug 15.Retrieved at http://www.icmr.ucsb.edu/programs/documents/Beasley.pdf

10. Bissonnet, P. (2015). A New Perspective on Advanced Space Travel. Journal of High Energy Physics, Gravitation and Cosmology, 1, 14-24. See also doi: 10.4236/jhepgc.2015.11002.

11. Lavenda, B.H. (2012). Twisted Tales from String Theory. Hadronic Journal, 35, 11-33.

12. Bissonnet, P. (2017).A Short Monograph on Lightning and the Earth's Changing Magnetic Field.Retrieved fromhttp://vixra.org/abs/1706.0481

13. Ball, Philip,The Mystery of What Causes Lightning. BBC "Future".Retrieved from http://www.bbc.com/future/story/20120926-what-causes-lightening

14. Loutre, M.F. (2003). Ice Ages (Milankovitch Theory), Elsevier Science, 995-1003.Retrieved from http://curry.eas.gatech.edu/Courses/6140/ency/Chapter10/Ency_Atmos/Ice_age.pdf

15. Colinvaux, P. (2007).Amazon Expeditions: My Search for the Ice-Age Equator, New Haven \& London: Yale University Press p. 235. 
